\title{
TOPOLOGY, INTERSECTIONS AND FLAT MODULES
}

\author{
CARMELO A. FINOCCHIARO AND DARIO SPIRITO \\ (Communicated by Irena Peeva)
}

\begin{abstract}
It is well known that, in general, multiplication by an ideal $I$ does not commute with the intersection of a family of ideals, but that this fact holds if $I$ is flat and the family is finite. We generalize this result by showing that finite families of ideals can be replaced by compact subspaces of a natural topological space, and that ideals can be replaced by submodules of an epimorphic extension of a base ring. As a particular case, we give a new proof of a conjecture by Glaz and Vasconcelos.
\end{abstract}

\section{INTRODUCTION}

Let $D$ be an integral domain with quotient field $K$. An overring of $D$ is a ring between $D$ and $K$. The set of all overrings of $D$ is denoted by $\operatorname{Over}(D)$, and can be endowed with a natural topology (called the Zariski topology) whose basis of open sets consists of the sets of the form

$$
\mathcal{B}\left(x_{1}, \ldots, x_{n}\right):=\left\{T \in \operatorname{Over}(D): x_{1}, \ldots, x_{n} \in T\right\},
$$

as $x_{1}, \ldots, x_{n}$ vary in $K$. Under this topology, $\operatorname{Over}(D)$ is a compact $T_{0}$ space with a unique closed point ( $D$ itself) and a generic point (the quotient field $K$ ). One of the clues that this topology is the most natural to be put on $\operatorname{Over}(D)$ is that it makes the localization map

$$
\begin{aligned}
\lambda: \operatorname{Spec}(D) & \longrightarrow \operatorname{Over}(D) \\
P & \longmapsto D_{P}
\end{aligned}
$$

a topological inclusion [4, Lemma 2.4].

This topology, whose origins can be traced back to Zariski's study of the space $\operatorname{Zar}(D)$ of the valuation overrings of an integral domain $D$ [25, Chapter $6, \S 17]$ (what is now called the Zariski space or the Riemann-Zariski space of D), has recently been studied in greater detail (see for example [7,8, 20,21]). For example, it has been proved that $\operatorname{Over}(D)$ is a spectral space, meaning that there is a ring $R$ such that $\operatorname{Spec}(R)$ is homeomorphic to $\operatorname{Over}(D)$ [5, Proposition 3.5]; the same can be proved of several distinguished subspaces of Over $(D)$, like for example local overrings [7, Corollary 2.14] or integrally closed overrings [5, Proposition 3.6].

The aim of this paper is to prove a simple and very general result (Theorem 3. in the form of Corollary (5) which intertwines the Zariski topology on Over $(D)$ with the algebraic properties of the overrings, namely the possibility to commute

Received by the editors October 1, 2015 and, in revised form, December 10, 2015.

2010 Mathematics Subject Classification. Primary 13A15, 13A18, 13C11.

Key words and phrases. Zariski topology, overrings, flat ideals. 
intersections and products in the case of compact spaces of overrings. In this way, we generalize [13, Lemma 1.1] (which deals with locally finite intersections) and [21, Theorem 3.5] (which proves the same for Noetherian collections of integrally closed overrings). As a consequence, we obtain a new proof of the Graz-Vasconcelos conjecture [12, page 340], independent from the one obtained in [23]. Since it poses no additional challenge, we also work in a more general setting, substituting to the extension $D \subseteq K$ any ring extension that is also an epimorphism, and using modules instead of only overrings.

\section{Results}

Let $A \subseteq B$ be a ring extension; we denote by $\mathcal{F}(B \mid A)$ the collection of all the $A$ submodules of $B$. The set $\mathcal{F}(B \mid A)$ becomes a $\mathrm{T}_{0}$ topological space by declaring, as a basis of open sets, the family of the sets of the form $\mathcal{B}\left(x_{1}, \ldots, x_{n}\right):=\{G \in \mathcal{F}(B \mid A)$ : $\left.x_{1}, \ldots, x_{n} \in G\right\}$, for $x_{1}, \ldots, x_{n}$ varying in $B$. Note that, since $\mathcal{B}\left(x_{1}, \ldots, x_{n}\right)=$ $\mathcal{B}\left(x_{1}\right) \cap \cdots \cap \mathcal{B}\left(x_{n}\right)$, a convenient subbasis for this topology is $\{\mathcal{B}(x): x \in B\}$. We call this topology the Zariski topology, as it generalizes the Zariski topology on $\operatorname{Over}(D)$ defined in the Introduction. Note that, in particular, the set $\mathcal{I}(A)$ of all the integral ideals of $A$ becomes then a subspace of $\mathcal{F}(B \mid A)$. On the $\operatorname{set} \operatorname{Spec}(A)$ of the prime ideals of $A$, this topology does not coincide with the classical Zariski topology, but rather with the so-called inverse topology (see [14] and the discussion before Example 2.2 of [22]). This should, however, not cause any confusion; the only place where we will consider $\operatorname{Spec}(R)$ will be Proposition 11.

If $X$ is any topological space and $Y \subseteq X$, we will denote by $\bar{Y}$ the closure of $Y$ in $X$.

Remark 1. Let $A \subseteq B$ be a ring extension and let $\mathcal{F}(B \mid A)$ be endowed with the Zariski topology. The following properties hold:

(1) For any $F, G \in \mathcal{F}(B \mid A)$, we have $F \in \overline{\{G\}}$ if and only if $F \subseteq G$.

(2) Any compact nonempty subspace $C$ of $\mathcal{F}(B \mid A)$ has minimal elements, with respect to the inclusion $\subseteq$. As a matter of fact, by Zorn's lemma it is enough to show that any chain (under inclusion) $\Sigma \subseteq C$ has a lower bound. By (1) the collection of sets $\mathcal{F}:=\{\overline{\{F\}} \cap C: F \in \Sigma\}$ is a chain. Thus, in particular, given any finite subset $F_{1}, \ldots, F_{n} \in \Sigma$, if $G$ is contained in all $F_{i}$, then $G \in \bigcap_{i=1}^{n} \overline{\left\{F_{i}\right\}} \cap C$. This proves that $\mathcal{F}$ is a collection of closed sets of $C$ with the finite intersection property. By compactness, there exists a submodule $F^{\star} \in \overline{\{F\}} \cap C$, for any $F \in \Sigma$, and applying again (1) we see that $F^{\star}$ is a lower bound of $\Sigma$ in $C$.

Now let $\phi: A \longrightarrow B$ be a ring homomorphism. Then, $\phi$ is an epimorphism in the category of rings if, for every $\psi_{1}, \psi_{2}: B \longrightarrow C$, the equality $\psi_{1} \circ \phi=\psi_{2} \circ \phi$ implies that $\psi_{1}=\psi_{2}$. If the inclusion map $A \hookrightarrow B$ is an epimorphism, we will call the ring extension $A \subseteq B$ an epimorphic extension.

Examples of epimorphisms are surjective maps and localizations; more generally, a map $\phi: A \longrightarrow B$ such that the induced homomorphism $\phi_{\mathfrak{p}}: A_{\mathfrak{p}} \longrightarrow B_{\mathfrak{p}}$ is surjective for every $\mathfrak{p} \in \operatorname{Spec}(A)$ such that $\phi(\mathfrak{p}) B \neq B$ is an epimorphism (maps with this property are called weakly surjective [16. Chapter $1, \S 3]$; on extensions, being an epimorphism and being weakly surjective are equivalent conditions [16, Theorem 4.4]). In particular, if $D$ is an integral domain and $K$ is its quotient field, the 
ring extension $D \subseteq K$ is an epimorphic extension. On the other hand, if $X$ is an indeterminate over $A$, then the extension $A \subseteq A[X]$ is not epimorphic: indeed, for every $\alpha \in A$, we can build a ring homomorphism $\psi_{\alpha}: A[X] \longrightarrow A$ by defining $\psi_{\alpha}(a):=a$ if $a \in A$ and $\psi_{\alpha}(X)=\alpha$. In this case, we have $\psi_{\alpha} \neq \psi_{\beta}$ if $\alpha \neq \beta$, but every $i \circ \psi_{\alpha}$ is the identity on $A$.

The first step of our way is the following fact, which is a generalization of 1 , Theorem 2].

Proposition 2. Let $A \subseteq B$ be an epimorphic extension. Let $I$ be a flat $A$ submodule of $B$, and let $G_{1}, \ldots, G_{n} \in \mathcal{F}(B \mid A)$. Then,

$$
I\left(G_{1} \cap \ldots \cap G_{n}\right)=I G_{1} \cap \ldots \cap I G_{n} .
$$

Proof. With a small abuse of notation, for any $F, G \in \mathcal{F}(B \mid A)$, we will denote by $F \otimes G$ the submodule of $B \otimes B$ generated by the elements $f \otimes g$, as $f$ varies in $F$ and $g$ varies in $G$. By induction, it suffices to show the statement for $n=2$. Consider the map

$$
\begin{aligned}
\lambda: B \otimes_{A} B & \longrightarrow B \\
b_{1} \otimes b_{2} & \longmapsto b_{1} b_{2} .
\end{aligned}
$$

Clearly, if $I, G \in \mathcal{F}(B \mid A)$, then $\lambda(I \otimes G)=I G$; therefore, by [18, Theorem 7.4]

$$
I\left(G_{1} \cap G_{2}\right)=\lambda\left(I \otimes\left(G_{1} \cap G_{2}\right)\right)=\lambda\left(\left(I \otimes G_{1}\right) \cap\left(I \otimes G_{2}\right)\right) .
$$

Since $A \subseteq B$ is an epimorphic extension, $\lambda$ is an isomorphism (indeed, this property actually characterizes epimorphisms [17, Lemma 1.0]); in particular, $\lambda$ is a bijection, and thus

$$
\lambda\left(\left(I \otimes G_{1}\right) \cap\left(I \otimes G_{2}\right)\right)=\lambda\left(I \otimes G_{1}\right) \cap \lambda\left(I \otimes G_{2}\right)=I G_{1} \cap I G_{2} .
$$

This completes the proof.

Note that this proposition does not hold if $A \subseteq B$ is not an epimorphism: for example, if $X$ is an indeterminate over $A, B=A[X]=I, G_{1}=A, G_{2}=X A[X]$, then $G_{1} \cap G_{2}=(0)$ and so $I\left(G_{1} \cap G_{2}\right)=(0)$, while $I G_{1} \cap I G_{2}=A[X] \cap X A[X]=$ $X A[X]$.

Theorem 3. Let $A \subseteq B$ be an epimorphic extension, let $I$ be a flat $A$-submodule of $B$ and let $Y$ be a (nonempty) compact subspace of $\mathcal{F}(B \mid A)$. Then, the following equality holds:

$$
I\left(\bigcap_{J \in Y} J\right)=\bigcap_{J \in Y} I J .
$$

Proof. The $(\subseteq)$ containment is obvious. Now take an element $x \in \bigcap\{I J: J \in Y\}$. For any $J \in Y$, by definition, there exist a positive integer $n_{J}$ and elements $i_{1}^{(J)}, \ldots, i_{n_{J}}^{(J)} \in I, t_{1}^{(J)}, \ldots, t_{n_{J}}^{(J)} \in J$ such that

$$
x=i_{1}^{(J)} t_{1}^{(J)}+\cdots+i_{n_{J}}^{(J)} t_{n_{J}}^{(J)}=\sum_{h=1}^{n_{J}} i_{h}^{(J)} t_{h}^{(J)} .
$$

Consider the open neighborhood $\Omega_{J}:=\mathcal{B}\left(\left\{t_{1}^{(J)}, \ldots, t_{n_{J}}^{(J)}\right\}\right)$ of $J$. Then the collection of sets $\mathscr{A}:=\left\{\Omega_{J}: J \in Y\right\}$ is an open cover of $Y$. By compactness, $\mathscr{A}$ admits a finite 
subcover, say $\left\{\Omega_{J_{1}}, \ldots, \Omega_{J_{r}}\right\}$, for suitable $J_{1}, \ldots, J_{r} \in Y$. For any $l=1, \ldots, r$, set $Y_{l}:=\Omega_{J_{l}} \cap Y$. By Proposition 2, we have

$$
I\left(\bigcap_{J \in Y} J\right)=I\left(\bigcap_{J \in Y_{1}} J \cap \ldots \cap \bigcap_{J \in Y_{r}} J\right)=I\left(\bigcap_{J \in Y_{1}} J\right) \cap \ldots \cap I\left(\bigcap_{J \in Y_{r}} J\right),
$$

and thus it suffices to show that $x \in I\left(\bigcap_{J \in Y_{l}} J\right)$, for each $l=1, \ldots, r$. However, the elements $t_{1}^{\left(J_{l}\right)}, \ldots, t_{n_{J_{l}}}^{\left(J_{l}\right)}$ belong to $J$ for every $J \in Y_{l}$, and thus they belong to the intersection $\bigcap\left\{J: J \in Y_{l}\right\}$; hence, the representation $x=\sum_{h=1}^{n_{J_{l}}} i_{h}^{\left(J_{l}\right)} t_{h}^{\left(J_{l}\right)}$ shows that $x \in I\left(\bigcap_{J \in Y_{l}} J\right)$.

Before giving some corollaries of independent interest, we state the following useful lemma.

Lemma 4. Let $A \subseteq B$ be a ring extension and let $\mathcal{F}(B \mid A)$ be endowed with the Zariski topology. Fix a submodule $I \in \mathcal{F}(B \mid A)$. Then, the maps

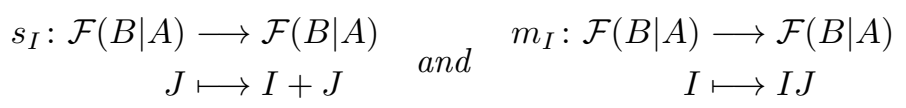

are continuous.

Proof. Let $\mathcal{B}(x)$ be a subbasic open set of $\mathcal{F}(B \mid A)$, with $x \in B$. If $J_{0} \in s_{I}^{-1}(\mathcal{B}(x))$, then $x=i+j$ for some $i \in I, j \in J_{0}$; therefore, $\mathcal{B}(j)$ is an open neighborhood of $J_{0}$ contained in $s_{I}^{-1}(\mathcal{B}(x))$, and thus $s_{I}$ is continuous. Similarly, if $J_{0} \in m_{I}^{-1}(\mathcal{B}(x))$, then $x=i_{1} j_{1}+\cdots+i_{n} j_{n}$ for some $j_{1}, \ldots, j_{n} \in J_{0}$ and $i_{1}, \ldots, i_{n} \in I$. Then, $J_{0} \in \mathcal{B}\left(j_{1}, \ldots, j_{n}\right) \subseteq m_{I}^{-1}(\mathcal{B}(x))$, and this shows that $m_{I}$ is continuous.

Note that the continuity of $m_{I}$ makes it possible to shorten the proof of [21, Lemma 3.7].

Corollary 5. Let $D$ be an integral domain, let $I$ and $T$ be $D$-submodules of the quotient field $K$ of $D$, and let $\Delta$ be a compact subset of $\operatorname{Over}(D)$, with respect to the Zariski topology. If $T$ is flat over $D$, then

$$
\left(\bigcap_{U \in \Delta} I U\right) T=\bigcap_{U \in \Delta}(I U T)
$$

Proof. By Lemma 4, the collection $\{I U: U \in \Delta\}$ is compact, since it is the continuous image of $\Delta$ via $m_{I}$. The conclusion is now an immediate consequence of Theorem 3 .

As a particular case of the main results, we now provide a new topological proof of the Glaz-Vasconcelos conjecture.

Corollary 6 ([23, Theorem 1.7]). Let D be an integrally closed integral domain, and let $I$ be a D-submodule of its quotient field $K$. If $I$ is flat over $D$, then $I=$ $\bigcap\{I V: V \in \operatorname{Zar}(D)\}$. 
Proof. The space Zar $(D)$ is compact in the Zariski topology [25, Chapter 6, Theorem 40]; moreover, since $D$ is integrally closed, $D=\bigcap\{V: V \in \operatorname{Zar}(D)\}$ [3, Corollary 5.22]. Hence, by Theorem 3 ,

$$
I=I D=I\left(\bigcap_{V \in \operatorname{Zar}(D)} V\right)=\bigcap_{V \in \operatorname{Zar}(R)} I V,
$$

as claimed.

Another immediate consequence of the main results deals with intersections of localizations of integral domains.

Corollary 7. Let $D$ be an integral domain, let $Y$ be a compact nonempty subspace of $\operatorname{Over}(D)$ such that $D=\bigcap\{R: R \in Y\}$, and let $S$ be a multiplicative subset of D. Then, $S^{-1} D=\bigcap\left\{S^{-1} R: R \in Y\right\}$.

Proof. It suffices to use Theorem 3 , keeping in mind that $S^{-1} D$ is a flat $D$-module.

Corollary 8 ([11, Proposition 43.5]). Let $D$ be an integral domain, let $Y$ be a locally finite subspace of $\operatorname{Over}(D)$ (i.e., any nonzero element of $D$ is noninvertible only in finitely many members of $Y$ ) such that $D=\bigcap\{R: R \in Y\}$, and let $S$ be a multiplicative subset of $D$. Then, $S^{-1} D=\bigcap\left\{S^{-1} R: R \in Y\right\}$.

Proof. By Corollary 7 , it is enough to show that a locally finite collection of overrings of $D$ is compact, with respect to the Zariski topology of $\operatorname{Over}(D)$.

Let $\mathscr{A}$ be an open cover of $Y$. By Alexander's Subbasis Theorem (see e.g. [15, Chapter 5, Theorem 6, page 139]), we can assume, without loss of generality, that $\mathscr{A}$ consists of subbasic open sets of $\operatorname{Over}(D)$, say $\mathscr{A}=\left\{\mathcal{B}\left(\frac{a_{i}}{b_{i}}\right): i \in I\right\}$, where $a_{i}, b_{i} \in D, b_{i} \neq 0$, for any $i \in I$. Now fix an index $i^{\prime} \in I$ and note that, by assumption, the set $Y^{\prime}:=\left\{R \in Y: b_{i^{\prime}}^{-1} \notin R\right\}$ is finite, say $Y^{\prime}=\left\{R_{1}, \ldots, R_{n}\right\}$. Thus, any member of $Y-Y^{\prime}$ belongs to $\mathcal{B}\left(\frac{a_{i^{\prime}}}{b_{i^{\prime}}}\right)$ and any $R_{j} \in Y^{\prime}$ belongs to some $\mathcal{B}\left(\frac{a_{i_{j}}}{b_{i_{j}}}\right)$. The proof is now complete.

Note that the main part of the proof of the previous corollary is also a consequence of [8, Proposition 2.9], where it was proved in the more general context of semistar operations; we inserted the proof here for the reader's convenience. Moreover, the proof of the previous corollary also extends [9] Remark 4.7], where the authors proved that any locally finite family of localizations is compact.

Corollary 9. Let $D$ be a Prüfer domain with quotient field $K$, let $\mathfrak{a}$ be an ideal of $D$, and let $Y \subseteq \mathcal{I}(D)$ be compact. Then,

$$
\mathfrak{a}+\bigcap_{\mathfrak{b} \in Y} \mathfrak{b}=\bigcap_{\mathfrak{b} \in Y}(\mathfrak{a}+\mathfrak{b})
$$

Proof. It suffices to prove that, for every prime ideal $\mathfrak{p}$, the equality

$$
\mathfrak{a} D_{\mathfrak{p}}+\left(\bigcap_{\mathfrak{b} \in Y} \mathfrak{b}\right) D_{\mathfrak{p}}=\left(\bigcap_{\mathfrak{b} \in Y}(\mathfrak{a}+\mathfrak{b})\right) D_{\mathfrak{p}}
$$


holds. Fix thus a prime ideal $\mathfrak{p}$, and let $V:=D_{\mathfrak{p}}$; since $D$ is a Prüfer domain, $V$ is a valuation domain.

Since $V$ is flat over $D$ and $\{\mathfrak{a}+\mathfrak{b}: \mathfrak{b} \in Y\}$ is compact (Lemma 4), we have, by Theorem 3

$$
\left(\bigcap_{\mathfrak{b} \in Y}(\mathfrak{a}+\mathfrak{b})\right) V=\bigcap_{\mathfrak{b} \in Y}((\mathfrak{a}+\mathfrak{b}) V)=\bigcap_{\mathfrak{b} \in Y}(\mathfrak{a} V+\mathfrak{b} V) .
$$

Now observe that, since $V$ is a valuation domain, the collection of ideals $Y^{\prime}:=\{\mathfrak{b} V$ : $\mathfrak{b} \in Y\}$ is totally ordered and compact, by Lemma 4. Thus, since by compactness $Y^{\prime}$ has minimal elements under inclusion (Remark 1), it follows that $Y^{\prime}$ has a minimum. Then, there is an ideal $\mathfrak{b}_{0} \in Y$ such that $\mathfrak{b}_{0} V \subseteq \mathfrak{b} V$, for any $\mathfrak{b} \in Y$. It follows that the last member of the equality (1) becomes

$$
\bigcap_{\mathfrak{b} \in Y}(\mathfrak{a} V+\mathfrak{b} V)=\mathfrak{a} V+\mathfrak{b}_{0} V=\mathfrak{a} V+\left(\bigcap_{\mathfrak{b} \in Y} \mathfrak{b} V\right)=\mathfrak{a} V+\left(\bigcap_{\mathfrak{b} \in Y} \mathfrak{b}\right) V,
$$

where the last equality is again a consequence of Theorem 3 The proof is now complete.

Remark 10. The previous corollary is closely related to the dual AB- $5 *$ of Grothendieck AB-5 (see, for example, 2]). Precisely, if $D$ is a Prüfer domain and any filter base of ideals of $D$ is compact, with respect to the Zariski topology of $\mathcal{I}(D)$, then $D$ is $\mathrm{AB}-5^{\star}$ (as $D$-module).

In the case of Prüfer domains, we can also prove a partial converse of Theorem 3. Recall that a prime ideal $P$ of a Prüfer domain $D$ is branched if the set of prime ideals of $D$ properly contained in $P$ has a maximum (see e.g. [11, Theorem 17.3]). If the dimension of $D$ is finite, every prime ideal is branched.

Proposition 11. Let $D$ be a Prüfer domain with quotient field $K$, and let $\Delta \subseteq$ $\operatorname{Spec}(D)$ be a nonempty set.

(a) $\Delta$ is compact (in the "classical" Zariski topology of $\operatorname{Spec}(D)$ ) if and only if, for every flat D-submodule $I$ of $K, \bigcap_{\mathfrak{p} \in \Delta} I D_{\mathfrak{p}}=I\left(\bigcap_{\mathfrak{p} \in \Delta} D_{\mathfrak{p}}\right)$.

(b) Suppose that every prime ideal of $D$ is branched. Then, $\Delta$ is compact (in the "classical" Zariski topology of $\operatorname{Spec}(R))$, if and only if $\bigcap_{\mathfrak{p} \in \Delta} D_{\mathfrak{q}} D_{\mathfrak{p}}=$ $D_{\mathfrak{q}}\left(\bigcap_{\mathfrak{p} \in \Delta} D_{\mathfrak{p}}\right)$ for every $\mathfrak{q} \in \operatorname{Spec}(D)$.

Proof. In both points, one implication follows from Corollary 7 and the fact that the map $\lambda: \operatorname{Spec}(D) \longrightarrow \operatorname{Over}(D), P \mapsto D_{P}$, is a topological inclusion. Suppose $\Delta$ is not compact, and let $T:=\bigcap_{\mathfrak{p} \in \Delta} D_{\mathfrak{p}}$; note that, without loss of generality, we can suppose that $\Delta=\Delta^{\downarrow}=\{\mathfrak{q} \in \operatorname{Spec}(D): \mathfrak{q} \subseteq \mathfrak{p}$ for some $\mathfrak{p} \in \Delta\}$, since $\Delta$ is compact if and only if $\Delta^{\downarrow}$ is compact.

The set of prime ideals $\mathfrak{p}$ such that $\mathfrak{p} T \neq T$ is the image of $\operatorname{Spec}(T)$ under the canonical map $\operatorname{Spec}(T) \longrightarrow \operatorname{Spec}(D)$; since it contains $\Delta$, and $\Delta$ is not compact, 
it must also contain a prime ideal $\mathfrak{q} \notin \Delta$. Since $D$ is a Prüfer domain, $\mathfrak{q}$ is a flat $D$-module; however,

$$
\bigcap_{\mathfrak{p} \in \Delta} \mathfrak{q} D_{\mathfrak{p}}=\bigcap_{\mathfrak{p} \in \Delta} D_{\mathfrak{p}}=T \neq \mathfrak{q} T=\mathfrak{q}\left(\bigcap_{\mathfrak{p} \in \Delta} D_{\mathfrak{p}}\right),
$$

against the hypothesis. Therefore, part (a) is proved.

If every prime ideal of $D$ is branched, so is $\mathfrak{q}$; therefore, there is a prime ideal $\mathfrak{q}_{0}$ directly below $\mathfrak{q}$. No ideal $\mathfrak{p} \in \Delta$ contains $\mathfrak{q}$; therefore, $D_{\mathfrak{p}} D_{\mathfrak{q}} \supsetneq D_{\mathfrak{q}}$, and in particular $D_{\mathfrak{q}_{0}} \subseteq D_{\mathfrak{p}} D_{\mathfrak{q}}$. Hence,

$$
\bigcap_{\mathfrak{p} \in \Delta} D_{\mathfrak{q}} D_{\mathfrak{p}} \supseteq D_{\mathfrak{q}_{0}} \supsetneq D_{\mathfrak{q}}=D_{\mathfrak{q}} T=D_{\mathfrak{q}}\left(\bigcap_{\mathfrak{p} \in \Delta} D_{\mathfrak{p}}\right),
$$

against the hypothesis. Part (b) is proved.

Note that part (b) of the previous proposition does not hold without the hypothesis that the prime ideals are branched: indeed, if $V$ is a valuation domain with maximal ideal $\mathfrak{m}$ unbranched, and $\Delta:=\operatorname{Spec}(V) \backslash\{\mathfrak{m}\}$, then

$$
V_{\mathfrak{m}} V=V=\bigcap_{\mathfrak{p} \in \Delta} V_{\mathfrak{p}}=\bigcap_{\mathfrak{p} \in \Delta} V_{\mathfrak{p}} V_{\mathfrak{m}}
$$

despite $\Delta$ not being compact.

Another question arising from Theorem 3 is if the equality $I\left(\bigcap_{J \in Y} J\right)=\bigcap_{J \in Y} I J$, for all compact families $Y$ of submodules of an epimorphic extension $A \subseteq B$, implies that $I$ is flat. This is true if the base ring $A$ is a domain, but fails in general (see [1, Theorem 2] and the subsequent discussion).

Remark 12. While Theorem 3 is quite general, it may be in general hard, or at least not easy, to find examples of compact subspaces to which it can be applied, or to prove that a given family is actually compact.

Some examples can be constructed using the fact that, under the Zariski topology, $\mathcal{F}(B \mid A)$ is a spectral space, i.e., it is homeomorphic to the prime spectrum of a ring [22, Example 2.2(2)]. For example, it follows form Remark 11(1) and either [24, Proposition 2.3] or [19, Proposition 2.2] that a subset $Y$ of $\mathcal{F}(B \mid A)$ is compact if and only if every element of the closure of $Y$, with respect to the constructible topology, contains a point of $Y$, where the constructible topology on $\mathcal{F}(B \mid A)$ is the coarsest topology on $\mathcal{F}(B \mid A)$ for which any open and compact subspace of $\mathcal{F}(B \mid A)$ is both open and closed.

Another class of examples comes from the domination map $d: \operatorname{Zar}(A) \longrightarrow$ $\operatorname{Spec}(A)$ of the Zariski space of a domain $A$ (i.e., the set of valuation overrings of $A)$. For example, if $S$ is a compact subspace of $\operatorname{Spec}(A)$, then $d^{-1}(S)$ is compact, by [19, Proposition 2.2 and Lemma 2.7(3)].

\section{ACKNOWLEDGMENT}

The authors would like to thank the referee for helpful comments and suggestions. 


\section{REFERENCES}

[1] D. D. Anderson, On the ideal equation $I(B \cap C)=I B \cap I C$, Canad. Math. Bull. 26 (1983), no. 3, 331-332, DOI 10.4153/CMB-1983-054-3. MR703406

[2] Pham Ngoc Ánh, Dolors Herbera, and Claudia Menini, AB-5* and linear compactness, J. Algebra 200 (1998), no. 1, 99-117, DOI 10.1006/jabr.1997.7216. MR1603265

[3] M. F. Atiyah and I. G. Macdonald, Introduction to commutative algebra, Addison-Wesley Publishing Co., Reading, Mass.-London-Don Mills, Ont., 1969. MR.0242802

[4] David E. Dobbs, Richard Fedder, and Marco Fontana, Abstract Riemann surfaces of integral domains and spectral spaces (English, with Italian summary), Ann. Mat. Pura Appl. (4) 148 (1987), 101-115, DOI 10.1007/BF01774285. MR932760

[5] Carmelo Antonio Finocchiaro, Spectral spaces and ultrafilters, Comm. Algebra 42 (2014), no. 4, 1496-1508, DOI 10.1080/00927872.2012.741875. MR3169645

[6] Carmelo A. Finocchiaro, Marco Fontana, and K. Alan Loper, The constructible topology on spaces of valuation domains, Trans. Amer. Math. Soc. 365 (2013), no. 12, 6199-6216, DOI 10.1090/S0002-9947-2013-05741-8. MR 3105748

[7] Carmelo Antonio Finocchiaro, Marco Fontana, and Dario Spirito, New distinguished classes of spectral spaces: A survey, Proceedings of the Graz conference, to appear.

[8] Carmelo Antonio Finocchiaro and Dario Spirito, Some topological considerations on semistar operations, J. Algebra 409 (2014), 199-218, DOI 10.1016/j.jalgebra.2014.04.002. MR3198840

[9] Marco Fontana and James A. Huckaba, Localizing systems and semistar operations, NonNoetherian commutative ring theory, Math. Appl., vol. 520, Kluwer Acad. Publ., Dordrecht, 2000, pp. 169-197. MR.1858162

[10] Marco Fontana, James A. Huckaba, and Ira J. Papick, Prüfer domains, Monographs and Textbooks in Pure and Applied Mathematics, vol. 203, Marcel Dekker, Inc., New York, 1997. MR 1413297

[11] Robert Gilmer, Multiplicative ideal theory, Queen's Papers in Pure and Applied Mathematics, vol. 90, Queen's University, Kingston, ON, 1992. Corrected reprint of the 1972 edition. MR 1204267

[12] Sarah Glaz and Wolmer V. Vasconcelos, Flat ideals. II, Manuscripta Math. 22 (1977), no. 4, 325-341. MR 0472797

[13] William Heinzer and Jack Ohm, Noetherian intersections of integral domains, Trans. Amer. Math. Soc. 167 (1972), 291-308. MR0296095

[14] M. Hochster, Prime ideal structure in commutative rings, Trans. Amer. Math. Soc. 142 (1969), 43-60. MR.0251026

[15] John L. Kelley, General topology, Springer-Verlag, New York-Berlin, 1975. Reprint of the 1955 edition [Van Nostrand, Toronto, Ont.]; Graduate Texts in Mathematics, No. 27. MR0370454

[16] Manfred Knebusch and Digen Zhang, Manis valuations and Prüfer extensions. I, Lecture Notes in Mathematics, vol. 1791, Springer-Verlag, Berlin, 2002. A new chapter in commutative algebra. MR.1937245

[17] Daniel Lazard, Autour de la platitude (French), Bull. Soc. Math. France 97 (1969), 81-128. MR 0254100

[18] Hideyuki Matsumura, Commutative ring theory, Cambridge Studies in Advanced Mathematics, vol. 8, Cambridge University Press, Cambridge, 1986. Translated from the Japanese by M. Reid. MR 879273

[19] Bruce Olberding, Affine schemes and topological closures in the Zariski-Riemann space of valuation rings, J. Pure Appl. Algebra 219 (2015), no. 5, 1720-1741, DOI 10.1016/j.jpaa.2014.07.009. MR3299704

[20] Bruce Olberding, Overrings of two-dimensional Noetherian domains representable by Noetherian spaces of valuation rings, J. Pure Appl. Algebra 212 (2008), no. 7, 1797-1821, DOI 10.1016/j.jpaa.2007.11.008. MR2400744

[21] Bruce Olberding, Noetherian spaces of integrally closed rings with an application to intersections of valuation rings, Comm. Algebra 38 (2010), no. 9, 3318-3332, DOI 10.1080/00927870903114979. MR2724221

[22] Bruce Olberding, Topological aspects of irredundant intersections of ideals and valuation rings, Proceedings of the Graz conference, to appear.

[23] Giampaolo Picozza and Francesca Tartarone, Flat ideals and stability in integral domains, J. Algebra 324 (2010), no. 8, 1790-1802, DOI 10.1016/j.jalgebra.2010.07.021. MR2678822 
[24] Niels Schwartz and Marcus Tressl, Elementary properties of minimal and maximal points in Zariski spectra, J. Algebra 323 (2010), no. 3, 698-728, DOI 10.1016/j.jalgebra.2009.11.003. MR2574858

[25] Oscar Zariski and Pierre Samuel, Commutative algebra. Vol. II, Springer-Verlag, New YorkHeidelberg, 1975. Reprint of the 1960 edition; Graduate Texts in Mathematics, Vol. 29. MR.0389876

Dipartimento di Matematica e Fisica, Università degli Studi "Roma Tre", Largo San Leonardo Murialdo, 1, 00146 Roma, Italy

E-mail address: carmelo@mat.uniroma3.it

Current address: Institute of Analysis and Number Theory, Graz University of Technology, 8010 Graz, Steyrergasse 31/II, Austria

E-mail address: finocchiaro@math.tugraz.at

Dipartimento di Matematica e Fisica, Università degli Studi "Roma Tre", Largo San LeONARdo Murialdo, 1, 00146 Roma, Italy

E-mail address: spirito@mat.uniroma3.it 